9 Jenkins RJ, Ashby HB. Gilles de la Tourette's syndrome in identical twins. Arch Neurol 1983;40:249-51.

10 Pauls DL, Cohen DJ, Heimbuch RC, Detlor J, Kidd KK. The familial pattern and transmission of Tourette syndrome and multiple tics. Arch Gen Psychiatry 19 1981;38:1091-3.

Secretarial help by Mrs R Beyer is kindly acknowledged as is bibliographical assistance of Mrs P Diercks.

Accepted 22 May 1987

\section{Long-standing "stiff-man" syndrome: a par- ticular form of disseminated inflammatory CNS disease?}

Sir: In this journal we recently reported on neurophysiological and neuropharmacological findings in a patient with the stiff man syndrome followed over 20 years. ${ }^{1}$ This patient has now been re-examined and has a new latent paresis of the left leg with slight lateralization of the deep tendon reflexes, but normal plantar responses. Multimodality evoked potentials were normal and reflex-electromyography demonstrated unchanged abnormal cutaneo-muscular reflexes. However, magnetic resonance imaging (MRI) revealed numerous small diameter $(<1 \mathrm{~cm})$ signal-enhancements at spin echo times of 60 and (less distinct) $120 \mathrm{~ms}$, located on both sides exclusively in the frontal and parietal white matter (fig left). In contrast to earlier examinations, moreover, evidence for local IgG-synthesis and positive oligoclonal IgG bands were found in the cerebrospinal fluid (CSF), associated with a normal cell count and total protein as well as albumin. Immunological investigations revealed an increased borrelia-IgG only in serum, but not in CSF.

Since 1979 we have followed another patient whose clinical picture and course resembles those of this patient; since 1978 she has suffered from severe stiffness of the trunk and proximal limb muscles which increases with exteroceptive stimuli. Stiffness is superimposed by acute and painful spontaneous myoclonic attacks of the trunk muscles. Muscle stiffness and spasms are completely abolished by diazepam or clonazepam. Symptoms return however, when this medication is interrupted. Repeated examinations of her CSF, cranial computed tomography, and multimodality evoked potentials have been normal. On recent examination, however, MRI showed periventricular white matter lesions (fig right), and oligoclonal banding was found in her CSF.

The fact that the diagnosis "stiff man" syndrome has to be revised (after a 10-20 years follow-up with numerous clinical, neurophysiological, CSF, and CCT-investigations) raises several questions. First of all, it underlines that such a (purely descriptive) diagnosis cannot be made appropriately on the basis of clinical and neurophysiological $Z$ data only: the wide variety of possible causes $\stackrel{\mathbb{C}}{=}$ of muscle stiffness have to be carefully searched for by means of repeated and $\subseteq$ exhaustive laboratory and neuroimaging methods.

In both cases, there was no evidence for immune vasculitis, and serological tests for $\frac{\rho}{\underline{2}}$ syphilis, toxoplasmosis, brucellosis, listeriosis, chlamydiasis as well as for various. neurotropic viruses were negative. Although both MRI and CSF findings (particularly in case 1) are suggestive of, they are certainly믈 not specific for multiple sclerosis. Moreover, $\bar{\sigma}$ not only the definite diagnosis but also the relationship between the different MRI abnormalities and the similar clinical findings in both cases remain obscure. How- $\infty$ ever, it seems likely that in both cases further $\overrightarrow{0}$ lesions exist within the brain-stem or spinal $\rightarrow$ cord, or both which escape our present $\vec{\omega}$ neuroimaging methods. Nevertheless, the question emerges, whether the stiff man syn- $\overline{\bar{z}}$ drome in other than these two cases is a0 particular manifestation of chronic inflam-cr matory CNS disorders such as multiple:sclerosis with abnormally slow progression.. Clinically, there is indeed a close $\vec{G}$ relationship between the "stiff man" sy용 drome and the syndrome of progress encephalomyelitis with rigidity. ${ }^{2}$

Among the relatively few reports in the literature which include CSF findings, on Maida et $^{3}{ }^{3}$ mention increased IgG sy thesis within the CNS. However, alrea $\bar{y}$
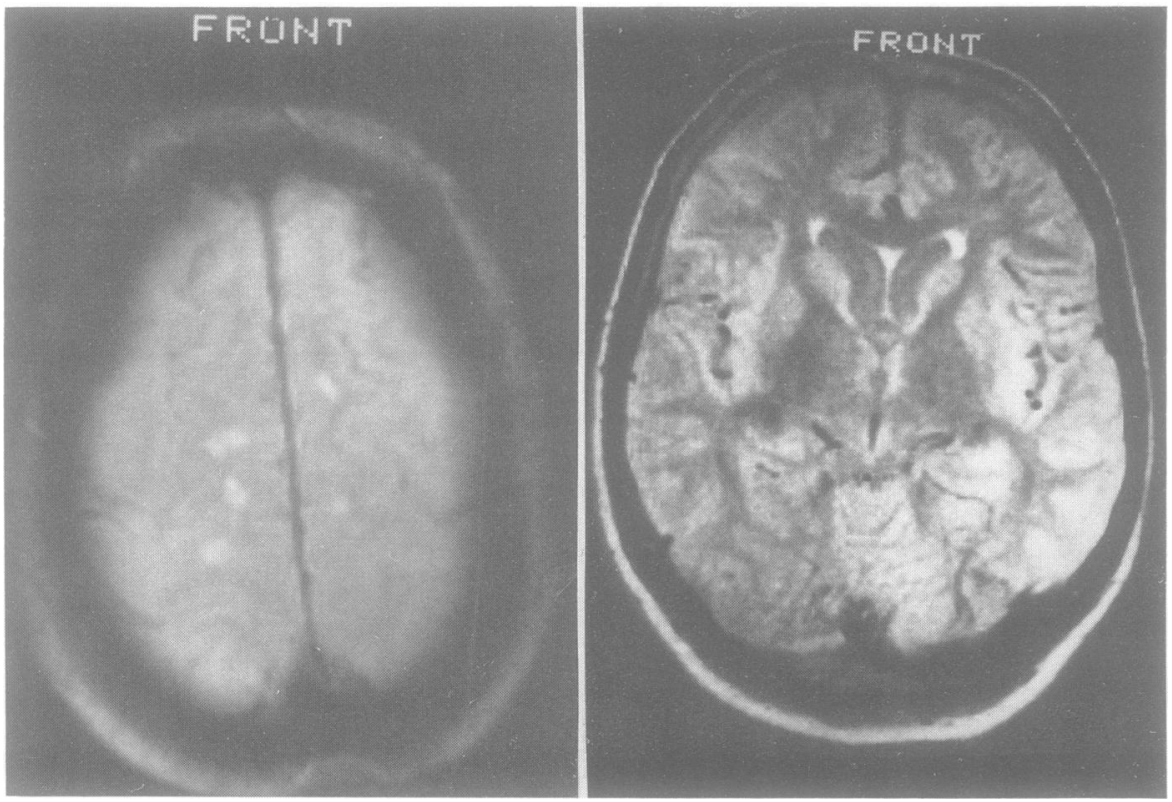

Fig Magnetic resonance imaging of two patients with the stiff-man syndrome. Note disseminated white matter lesions in case 1 (left) and signal enhancements around the ventral horns in case 2 (right). 
Moersch's and Woltman's ${ }^{4}$ Iowa farmer (case 1) had a Nonne-positive CSF suggesting an increased autochthonous IgG production. Abnormal CCT findings were seen in one patient with the "jerking stiff man syndrome", ${ }^{5}$ but not in a second one, ${ }^{6}$ and in one typical case, ${ }^{3}$ comprising marked cerebellar atrophy and an enlarged fourth ventricle or enlarged basal cysterns, hypodense areas in the temporal lobes, and dilatation of the interhemispherical fissure. ${ }^{3}$

H-M MEINCK

K RICKER*

Neurologische Klinik

Oberer Eselsberg 45

D-7900 Ulm, FRG

*Neurologische Klinik,

Kopfklinikum.

Josef-Schneider-Straße 11,

D-8700 Würzburg, FRG

Accepted 2 April 1987

References

I Meinck HM, Ricker K, Conrad B. The stiff-man syndrome: new pathophysiological aspects from abnormal exteroceptive reflexes and the response to clomipramine, clonidine and tizanidine. $J$ Neurol Neurosurg Psychiatry 1984:47:280-7.

2 Whiteley AM, Swash M, Urich H. Progressive encephalomyelitis with rigidity. Brain 1976;99:27-42.

3 Maida E, Reisner T, Summer K, SandorEggerth H. Stiff-man syndrome with abnormalities in CSF and computerized tomography findings. Arch Neurol 1980;37:182-3.

4 Moersch FP, Woltman HW. Progressive fluctuating muscular rigidity and spasm ("stiff-man" syndrome): report of a case and some observations in 13 other cases. Mayo Clin Proc 1956;31:421-7.

5 Leigh PN, Rothwell JC, Traub M, Marsden CD. A patient with reflex myoclonus and muscle rigidity: "jerking stiff-man syndrome". J Neurol Neurosurg Psychiatry 1980:43:1125-31.

6 Alberca R, Romero M, Chaparro J. Jerking stiff-man syndrome. J Neurol Neurosurg Psychiatry 1982;45:1159-60.

The Miller Fisher syndrome following campylobacter enteritis: a report of two cases

The Miller Fisher syndrome is a rare variant of post infectious polyneuritis in which opthalmoplegia, areflexia and ataxia occur. We report two cases associated with campylobacter infection.

A 37 year old man had a 2 day history of diarrhoea 7 days after returning from hol- iday in Tenerife. A week later he noted parasthesiae in his hands and feet, followed over the next 5 days by diplopia, nasal regurgitation of fluids and difficulty in walking. There was no relevant past history. Systems enquiry was unhelpful. On examination he was apyrexial. The pulse rate was 80 per minute, the blood pressure $130 / 80 \mathrm{mmHg}$. There was complete bilateral external opthalmoplegia. The pupils were dilated with a sluggish response to light and accommodation; the fundi were normal. Bilateral lower motor neuron facial nerve palsies, absent gag reflexes, paralysis of the soft palate and bulbar dysarthria were noted. Muscle bulk, tone and power were normal apart from minimal weakness in the long extensors of his fingers. All deep tendon reflexes were absent with flexor plantar responses. The abdominal reflexes were retained. There was no sensory abnormality; proprioception and vibration were normal. Formal tests of coordination in the limbs were well performed but he demonstrated considerable ataxia of stance and gait. There were no other signs. Haematological and biochemical investigations were normal. There were no cells in the cerebrospinal fluid; the protein concentration was $0.54 \mathrm{~g} / \mathrm{l}$ and the glucose $4 \mathrm{mM} / \mathrm{l}(73 \mathrm{mg} / \mathrm{dl})$. Nerve conduction studies and computed tomography of his posterior fossa were normal. Campylobacter jejuni, sensitive to erythromycin, was grown from his stool. There was no other evidence of infection. A 5 day course of erythromycin cleared his stool of the organism. $\mathrm{He}$ required nasogastric feeding but did not suffer any respiratory embarassment. His neurological disability remained static for 10 to 14 days and thereafter rapidly improved. No definitive treatment other than the antibiotic was employed. Three months later he has returned to normal with no residual signs.

A 28 year old man had a 3 day episode of diarrhoea on holiday in Portugal. Ten days later he developed diplopia and parasthesiae over the anterior chest. His past history was unremarkable. On examination he was apyrexial. The pulse rate was 76 per minute, blood pressure $120 / 80 \mathrm{mmHg}$. There were bilateral sixth nerve palsies and impaired upward gaze. There were no other cranial nerve abnormalities. Muscle bulk and tone were normal. There was grade $4+/ 5$ weakness of shoulder abduction, wrist movements and finger extension bilaterally. Deep tendon reflexes in the arms were absent. Both knee and ankle jerks were present with flexor plantar responses. No sensory abnormality was detected; proprioception and vibration were preserved. No formal limb ataxia could be demonstrated, but he had ataxia of tandem gait. The rest of the examination was normal. Haematological and biochemical investigations were normal. The cerebrospinal fluid protein concentration was $0.36 \mathrm{~g} / \mathrm{l}$, the glucose $3.5 \mathrm{mM} / \mathrm{l}(64 \mathrm{mg} / \mathrm{dl})$; there were no cells on microscopy. Stool culture grew Campylobacter jejuni. Campylobacter antibody titres were $1 / 64$ (IgG 2.9 units, IgM 4.1 units of optical density) indicating a recent infection. ${ }^{2}$ Titres to parainfluenza fell from $1 / 128$ to $1 / 64$ in 7 days. There was no other evidence of infection. Nerve conduction studies (including " $F$ " waves), electroencephalography and computed tomography of his posterior fossa were normal. His condition improved over a period of 10 days without specific treatment. There were no respiratory or bulbar symptoms at any time. He remains well 18 months later.

There has been one report of Miller Fisher syndrome presenting with campylobacter enteritis. ${ }^{3}$ We report a further two cases associated with this organism. The neurological illness in these patients was preceded by a short episode of diarrhoea but there were no symptoms of enteritis as presentation. One patient received antibiotiळ్థ therapy but both made full recoveries wit领 윽 no other specific treatment. Retrospective evidence of campylobacter infection has $z$ been demonstrated in over a third of a recents series of patients with Guillain-Barré syno drome. $^{4}$ A history of diarrhoeal illness should be sought and asymptomatic camo pylobacter infection considered in patients presenting with these conditions. No comment can be made, however, on the possible efficacy of clearing the organism from the stool. There remains debate about the exact site of the lesions in Miller Fisher syndrome. ${ }^{5}$ The cranial nerve palsies and ataxia indicate brainstem pathology but the areflexia points to a peripheral component as well. The recognition of this syndrome is important as in spite of its alarming nature the course and outcome warrant an optimistic outlook.

TIM ROBERTS
AJAY SHAH
JG GRAHAM
INF MCQUEEN
Department of Neurology,
University Hospital of Wales,
Heath Park, Cardiff CF4 $4 X N, U K$

References

${ }^{1}$ Fisher M. An Unusual Variant of acute idiopathic polyneuritis (syndrome of 